\title{
The classification of some spherically symmetric space-time metrics
}

\section{J.M. Foyster and C.B.G. Mclntosh}

It is shown that the Petrov-Plebanski classification of the trace-free Ricci tensors of some spherically symmetric metrics is invariant, contrary to an assertion by Takeno and Kitamura concerning these metrics.

In a discussion of spherically symetric space-time metrics, Takeno and Kitamura [4] assert that some metrics belong to either classes $[T-3 S]_{[1-1]}$ or $[3 T-S]_{[1-1]}$ in the Petrov-Plebanski classification of their trace-free Ricci tensors depending on the co-ordinate system in which the metric is expressed, that is, the class is not invariant. Their closing comment on their second example used to illustrate this assertion is "thus we find that the type of the eigenvalues of the [metric in the example] depends on the choice of the co-ordinate system".

It is shown in this paper that their two examples do not show such a breakdown of invariance. Their metrics which belong to classes [T-3S] or [3T-S] (se drop the [1-1] subscripts) do so only for different domains of their co-ordinates and only in the co-ordinate domain where these metrics are of type $[T-3 S]$ can real co-ordinate transformations be found which map the metrics into corresponding [T-3S] metrics. Thus the Ricci tensor type is invariant, provided that only real co-ordinate transformations are allowed (and these are the only ones with physical meaning).

Received 12 October 1972. 
The first example discussed by Takeno and Kitamura concerns the Einstein metric (or static Robertson-Walker metric)

$$
d \theta^{2}=d t^{2}-\left(1-r^{2} / R^{2}\right)^{-1} d r^{2}-r^{2} d \tau^{2}
$$

where

$$
d \Sigma^{2}=d \theta^{2}+\sin ^{2} \theta d \phi^{2}
$$

and $R^{2}$ is a constant (their Equation 9.1). The Ricci tensor type is $[T-3 S]$ as the authors show. They say that (1) can be transformed into

$$
d s^{2}=\frac{R^{2}}{4 u v}\left\{(u+v)^{2} d \Sigma^{2}-4 d u d v\right\}
$$

Here $u$ and $v$ are their $r$ and $-t$ respectively.

For $R^{2}>0$ the appropriate transformation is

$$
\begin{aligned}
r^{2} & =\frac{R^{2}(u+v)^{2}}{4 u v}, u v>0, \\
t & = \pm i \frac{R}{2} \ln (u v) .
\end{aligned}
$$

It is thus not possible to obtain (3) from (1) by a real co-ordinate transformation. For $R^{2}<0$, the appropriate transformation is

$$
\begin{aligned}
r^{2} & =\frac{R^{2}(u+v)^{2}}{4 u v} R^{2}<0, u v<0, \\
t & = \pm \frac{3}{2}\left(-R^{2}\right)^{\frac{1}{2}} \ln (-u v),
\end{aligned}
$$

which gives a real co-ordinate transformation from (1) to (3) and vice versa.

The Ricci tensor type for (3) is

$$
\begin{aligned}
& {[3 T-S] \text { for } R^{2}>0,} \\
& {[T-3 S] \text { for } R^{2}<0,}
\end{aligned}
$$

as Takeno and Kitamura point out. Thus it is only the $[T-3 S]$ metrics which map into each other.

Their second example concerns 


$$
d s^{2}=f(z)\left(d t^{2}-d r^{2}-r^{2} d \Sigma^{2}\right), \quad z \equiv t^{2}-r^{2} .
$$

The Ricci tensor class is

$$
\begin{aligned}
& {[3 T-S] \text { for } Z<0,} \\
& {[T-3 S] \text { for } Z>0 .}
\end{aligned}
$$

If $z>0$, there is a real co-ordinate transformation which maps (7) onto the class $[T-3 S]$ Robertson-Walker metric

$$
d s^{2}=d t^{2}-\frac{e^{2^{g(t)}}}{\left(1+r^{2} / 4 R^{2}\right)^{2}}\left(d r^{2}+r^{2} d r^{2}\right),
$$

with $R^{2}$ a non-zero constant. There is no such transformation when $z<0$.

Thus in these two examples the Ricci tensor classes remain invariant under real co-ordinate transformations in these cases and it is not possible to find a real mapping from a metric of one class to a metric of another class.

We find that the easiest way to calculate the relevant Ricci tensor class of a metric is to choose an appropriate orthonormal null tetrad for that metric, to calculate the spin coefficients for that metric as defined by Newman and Penrose [3] and then to examine the form of the non-zero $\Phi_{a b}$. Thus for the metric (3) a tetrad can be found such that the non-zero $\Phi a b$ and $\Lambda$ are

(a) $R^{2}<0, u>0, v<0$,

$$
\Phi_{00}=\Phi_{22}=2 \Phi_{11}=2 \Lambda=\frac{1}{2} R^{-2} \text {; }
$$

(b) $R^{2}>0, u>0, v<0$,

$$
\Phi_{00}=\Phi_{22}=-2 \Phi_{11}=-2 \Lambda=-\frac{3}{2} R^{-2} \text {. }
$$

The appropriate classes for these $\Phi_{a b}$ 's are then determined from the canonical forms

(a) $[T-3 S] \Phi_{00}, \Phi_{11}, \Phi_{22},>0,4 \Phi_{11}^{2}=\Phi_{00} \Phi_{22}$; 


$$
\begin{aligned}
& \text { (b) }[3 T-S] \Phi_{00}, \Phi_{11},>0, \Phi_{02} \neq 0,4 \Phi_{11}^{2}=\Phi_{02} \Phi_{20} \text { or } \\
& \Phi_{00},-\Phi_{11}, \Phi_{22},>0,4 \Phi_{11}^{2}=\Phi_{00} \Phi_{22} \text {; }
\end{aligned}
$$

(see Ludwig and Scanlan [1] and Lun and Mclntosh [2]).

For every [T-3S] metric which we have studied, there is a [3T-S] metric related to the first metric by a complex coordinate mapping and it seems to us that this is always so. We are unable, however, to offer any proof of this conjecture.

All metrics which are solutions of Einstein's field equations in the case of a perfect fluid are of class [T-3S]. We do not know the physical interpretation of [3T-S] metrics, other than ones which are solutions of $R_{a b}=-\phi, a^{\phi, b}$ where $\phi$ is a scalar function of spacelike coordinates such as those discussed by Lun and Mclntosh [2].

\section{References}

[1] Garry Ludwig and Gerry Scanlan, "Classification of the Ricci tensor", Comm. Math. Phys. 20 (1971), 291-300.

[2] A.W.-C. Lun and C.B.G. McIntosh, "Type $N$ solutions of the vacuum scalar-tensor field equations", General relativity and gravitation (to appear).

[3] Ezra Newman and Roger Penrose, "An approach to gravitational radiation by a method of spin coefficients", J. Mathematical Phys. 3 (1962), 566-578.

[4] Hyôitirô Takeno and Shin-ichi Kitamura, On the Einstein tensors of spherically symmetric space-times (RRK 69-13. Research Institute for Theoretical Physics, Hiroshima University, Hiroshima, 1969).

Australian Council for Educational Research, Department of Mathematics, Hawthorn, Monash University, Victoria; Clayton, Victoria. 